\title{
Correlação entre fadiga e desempenho ocupacional de indivíduos com esclerose múltipla ${ }^{1}$
}

\author{
Valéria Sousa de Andrade, Mayara Mirella Araújo Seabra, Isabella Elias de Moura Ramos \\ Universidade Federal do Triângulo Mineiro - UFTM, Uberaba, MG, Brasil.
}

\begin{abstract}
Resumo: Introdução: A esclerose múltipla (EM) é uma doença desmielinizante caracterizada por vários sintomas, dentre os quais a fadiga, manifestação que pode conduzir ao comprometimento do desempenho das atividades diárias e da qualidade de vida do indivíduo que a exibe. Objetivo: Identificar a correlação entre fadiga e desempenho ocupacional em atividades consideradas significativas por indivíduos com EM. Método: Indivíduos com EM que frequentavam o ambulatório de um hospital universitário compuseram a amostra. A "fadiga" e o "desempenho ocupacional" foram avaliados pela Escala de Severidade da Fadiga e pela Medida Canadense de Desempenho Ocupacional, respectivamente. Resultados: As atividades com maior grau de importância e de dificuldade na execução relatadas pelos participantes foram andar na rua, subir e descer escadas, e ir à casa de amigos. Através do Coeficiente de Correlação de Pearson, observou-se inexistência de relação estatisticamente significativa entre as variáveis "fadiga e desempenho na execução" das atividades (r: $-0,11)$ ou entre "fadiga e a satisfação ao desempenhar" aquelas tarefas (r: $-0,26)$. Por outro lado, constatou-se relação positiva estatisticamente significativa entre desempenho das atividades e satisfação ao desempenhá-las (r: 0,51). Conclusão: A não correlação entre as variáveis "fadiga e desempenho ocupacional" indicou ausência de interferência desse sintoma na realização das atividades consideradas importantes pelos participantes. A relação positiva estatisticamente significativa entre as variáveis "desempenho da atividade e satisfação ao desempenhá-la" ilustrou atitude positiva e otimista por parte dos participantes em relação ao modo como realizam suas atividades rotineiras.
\end{abstract}

Palavras-chave: Fadiga, Esclerose Múltipla, Atividades Cotidianas.

\section{Correlation between fatigue and occupational performance of people with multiple sclerosis}

\begin{abstract}
Introduction: Multiple sclerosis (MS) is a demyelinating disease characterized by many symptoms, including fatigue that may lead to the impairment on the performance of daily activities and quality of life of the subjects affected by the disease. Objective: Identify the relationship between fatigue and occupational performance on activities considered significant by individuals with MS. Methods: The sample consisted of subjects with MS that attended the outpatient clinic of a university hospital. The variables "fatigue" and "occupational performance" were evaluated by the Fatigue Severity Scale and the Canadian Occupational Performance Measure, respectively. Results: The activities with the highest score of importance and difficulty of performance were walking on the street, go up and down the stairs, and go to friend's house. Pearson correlation coefficient showed no statistically significant relationship between "fatigue and occupational performance" (r: - 0.11$)$ or "fatigue and the satisfaction" to perform the tasks (r: -26$)$. On the other hand, it was observed a statistically significant relationship between activity performance and satisfaction to accomplish it (r: 0.51). Conclusion: The absence of relationship between the variables "fatigue and occupational performance" indicated a non-interference of that symptom on the activities accomplishment. It was observed a statistically significant positive relationship between the variables "activity performance" and "satisfaction" which illustrated a positive and optimistic attitude by the participants in relation to the way they conduct their daily activities.
\end{abstract}

Keywords: Fatigue, Multiple Sclerosis, Daily Activities.

Autor para correspondência: Valéria Sousa de Andrade, Departamento de Terapia Ocupacional, Instituto de Ciências da Saúde, Universidade Federal do Triângulo Mineiro, Av. Getúlio Guaritá, 159, 3. ${ }^{\circ}$ andar, CEP 38025-440, Uberaba, MG, Brasil, e-mail: valeria-to@hotmail.com Recebido em Dez. 3, 2014; $1^{\text {a }}$ Revisão em Mar. 11, 2015; 2a Revisão em Jun.22, 2015; Aceito em Ago. 4, 2015. 


\section{Introdução}

A esclerose múltipla (EM) é uma doença neurológica autoimune, inflamatória e desmielinizante, que afeta prioritariamente mulheres entre 20 e 40 anos (SILVA; NASCIMENTO, 2014). Trata-se de uma doença lenta e progressiva, com remissão de surtos, apresentando-se de formas diferentes, a saber: a) recorrente remitente $(\mathrm{RR})$ - tipo mais frequente, no qual os sintomas se exacerbam por cerca de uma ou duas semanas, seguidos por uma melhora gradual. No início da doença, a recuperaçáo dos sintomas pode ser total durante o período de remissão, sendo observadas, contudo, repetiçóes das crises e consequente acúmulo de déficits; b) primariamente progressiva (PP) - ocorre em $10 \%$ a $20 \%$ dos pacientes, havendo comprometimento desde o surgimento do primeiro sintoma em estágios ou remissôes; c) secundariamente progressiva (SP) - acomete em torno de $90 \%$ das pessoas, iniciando como forma recorrente remitente, mas sendo evidenciado comprometimento progressivo sem fases de melhora dos sintomas; d) Forma progressiva com surtos - há progressão da doença desde o surgimento, sendo observados claros surtos de exacerbação dos sintomas com ou sem recuperação total das funções afetadas, e piora progressiva fora dos momentos de surto (BARROSO et al., 2013; SANTOS et al., 2010).

Atualmente, há aproximadamente 1,5 milhão de pessoas no mundo com EM (MILO; KAHANA, 2010). Mesquita (2013) afirma que são escassos estudos epidemiológicos sobre EM no Brasil, mas que há uma estimativa de 25 mil indivíduos com a doença, segundo a Multiple Sclerosis International Federation, estando a maioria deles localizada na Regiâo Sudeste.

Os sinais e sintomas mais comumente encontrados são fadiga, problemas de visão, tremor, disfunção intestinais e da bexiga, espasmos, alteração na fala, dificuldade de deglutição, déficits sexuais, dificuldade em executar as atividades cotidianas - como tomar banho, comer, vestir e cuidar dos afazeres domésticos -, distúrbios de locomoção, dor, empecilho de aprendizado e concentração, e depressão. Como consequência, a EM pode afetar a qualidade de vida dos indivíduos que a possuem (QUINTANILHA; LIMA, 2010).

Dentre os sintomas citados, a fadiga é particularmente um dos mais comuns, sendo relatada por $50 \%$ a $70 \%$ dos pacientes (LOPES et al., 2010). Esse sintoma se diferencia de todas as experiências de cansaço que indivíduos sem a doença já tenham possivelmente experimentado, pois a maioria daqueles com EM o considera como um dos mais incapacitantes. Além disso, os pacientes acreditam que a fadiga influencia fortemente o cotidiano e a qualidade de vida, conduzindo a alteraçóes no desempenho das atividades diárias (FLENSNER et al., 2013).

Segundo a Associação Canadense de Terapia Ocupacional, o termo 'desempenho ocupacional' (DO) é relatado como a interação dos fatores "pessoa" (composto por componentes físicos, afetivos, cognitivos e espirituais), "ambiente" (constituído por elementos físico, cultural, virtual e institucional) e "ocupação" (estruturado em autocuidado, produtividade e lazer) (MAGALHÃES; MAGALHÃES; CARDOSO, 2009). Esse conceito revela que dificuldades no DO ou nos níveis de satisfaçáo para com o mesmo podem ser ocasionados pelo desequilíbrio entre os fatores "pessoa, ambiente e ocupação” (OLIVEIRA et al., 2013).

A terapia ocupacional (TO) se apropria do modelo proposto pela Associação Canadense de Terapia Ocupacional ao focar suas intervençôes na interação entre pessoa, ambiente e ocupação, a fim de reabilitar indivíduos com EM (HOFFMANN; DYNIEWICZ, 2009). Ao fazê-lo, a TO visa prioritariamente a reduzir os efeitos das limitaçóes resultantes dessa doença e promover o máximo de independência, qualidade de vida e participação segura, funcional e confortável nas tarefas desempenhadas pelo indivíduo (COPPERMAN; FORWELL; HUGOS, 2008).

Com base no exposto, o presente estudo objetivou identificar a correlaçáo entre fadiga e DO em atividades consideradas significativas por indivíduos com EM.

\section{Materiais e métodos}

A pesquisa consiste em um estudo exploratório descritivo de corte transversal desenvolvida no Ambulatório de Esclerose Múltipla do Hospital Universitário localizado em uma cidade de porte médio do Triângulo Mineiro, Minas Gerais, durante o período de maio a setembro de 2011.

\subsection{Amostra}

Os indivíduos que compuseram a amostra do estudo foram selecionados por meio de amostragem por conveniência, tendo correspondido aos pacientes que frequentavam regularmente tal ambulatório. Foram elegíveis a participar do estudo indivíduos com idade igual ou superior a 18 anos que apresentavam diagnóstico de EM e que demonstravam preservação da capacidade cognitiva. Para apurar o nível da cognição dos participantes, foi utilizado o Miniexame do Estado Mental (MEEM), tendo sido excluídos indivíduos com escores inferiores a 13/14 para 
analfabetos e 23/24 para alfabetizados, segundo Kochahnn et al. (2010). Os dados sociodemográficos e clínicos dos participantes foram obtidos a partir de seus prontuários. A fim de obedecer à Resoluçáo n. ${ }^{\circ} 466 / 2012$ (BRASIL, 2013), a pesquisa foi submetida e aprovada pelo Comitê de Ética em Pesquisa em Seres Humanos da Universidade Federal do Triângulo Mineiro (parecer n. ${ }^{\circ}$ 1784/2011). Além disso, os participantes assinaram um Termo de Consentimento Livre e Esclarecido.

\subsection{Instrumentos da coleta dos dados}

A fadiga foi avaliada por meio da Escala de Severidade da Fadiga (ESF), protocolo composto por nove tópicos, que verificam a fadiga através de níveis de mensuração de 1 (menor nível de fadiga) a 7 (maior nível de fadiga). O escore total varia de 9 a 63 , sendo a pontuação superior a 27 considerada indicativa de presença de fadiga (NOGUEIRA et al., 2009). E ainda, conforme Mendes et al. (2000), de 28 a 39, fadiga leve; 40 a 51, fadiga moderada; 52 a 63, fadiga grave.

Para averiguar o DO dos participantes, utilizou-se a Medida Canadense do Desempenho Ocupacional (COPM). O instrumento permite ao individuo identificar suas dificuldades ao realizar atividades nas áreas de autocuidado, produtividade e lazer. Para condução do instrumento, o indivíduo inicialmente avalia a importância de até cinco atividades que considera dificultosas de realizar, utilizando-se, para tal, de uma escala de 1 (menor nível de importância) a 10 (maior nível de importância), para quantificá-las. Posteriormente, ele avalia o nível do seu desempenho para realizar tais atividades e sua satisfação ao desempenhá-las. Neste caso, uma escala de 1 a 10 é utilizada, correspondendo 1 a "grande dificuldade ou não satisfação", e 10 a "sem quaisquer dificuldades ou completa satisfação" (MAGALHÂES; MAGALHÃES; CARDOSO, 2009).

Os instrumentos foram utilizados uma vez, junto a cada participante. Visto não serem instrumentos autoaplicáveis, foram conduzidos pelos pesquisadores em contato direto com os participantes, sendo necessários em torno de 20 encontros para que todos pudessem respondê-los.

\subsection{Análise dos dados}

O conteúdo descritivo do estudo foi avaliado por meio de frequência relativa e absoluta. Para verificação da distribuição dos dados, utilizou-se o Teste de Kolmogorov-Smirnov, a partir do qual foi observada normalidade na mesma. A partir de tal dado, utilizou-se o Coeficiente de Correlaçáa de Pearson para avaliar a correlação entre fadiga e DO, tendo sido considerado nível de significância de $95 \%$. Os dados foram analisados pelo software estatístico SPSS (v. 20).

\section{Resultados}

Dos 29 pacientes considerados elegíveis, dois foram excluídos por não frequentarem o Ambulatório de Esclerose Múltipla durante o período da coleta de dados. Assim, a amostra final desse trabalho foi composta por 27 pacientes com EM.

Dezoito $(66,7 \%)$ indivíduos eram do sexo feminino e nove (33,3\%), do sexo masculino, variando suas idades entre 19 e 69 anos (média $=47$; DP $=10$ ). Considerando-se o nível de escolaridade, a maior parte da amostra $(10 / 38,5 \%)$ concluiu o Ensino Médio (Tabela 1).

O tempo de instalação da EM variou entre um e 42 anos, tendo $25(92,6 \%)$ dos participantes apresentado o tipo RR e dois $(7,4 \%)$, o tipo SP. De acordo com o prontuário dos pacientes, foram constatados entre um e nove surtos desde o início da doença. Ao serem questionados sobre a presença de fadiga, 22 (81,5\%) indivíduos afirmaram apresentarem-na, ao passo que cinco $(18,5 \%)$ não a observavam. Após a aplicação da ESF, verificou-se que três $(11,1 \%)$ indivíduos não possuíam fadiga e $24(88,9 \%)$ apresentaram esse sintoma em níveis diferentes. Dentre estes, sete $(29,2 \%)$ demonstraram possuir fadiga grave, $11(45,8 \%)$ fadiga moderada e seis $(25 \%)$ fadiga leve (Figura 1$)$.

Tabela 1. Características sociodemográficas da $\operatorname{amostra}(\mathrm{n}=27)$.

\begin{tabular}{lcc}
\hline \multicolumn{1}{c}{ Características } & $\mathbf{n}$ & $\%$ \\
\hline Sexo & & \\
$\quad$ Feminino & 18 & 66,7 \\
$\quad$ Masculino & 9 & 33,3 \\
Idade (anos) & & \\
$\quad<20$ & 1 & 3,7 \\
$21-40$ & 6 & 22,2 \\
$41-60$ & 16 & 59,3 \\
$>60$ & 4 & 14,8 \\
Escolaridade & & \\
$\quad$ Ensino Fundamental incompleto & 6 & 23 \\
Ensino Fundamental completo & 2 & 7,7 \\
Ensino Médio incompleto & 0 & 0 \\
Ensino Médio completo & 10 & 38,5 \\
Ensino Superior incompleto & 1 & 3,9 \\
Ensino Superior completo & 1 & 26,9 \\
\hline
\end{tabular}


De acordo com a COPM, os participantes enumeraram as ocupaçóes consideradas importantes para si próprios e com maior nível de dificuldade de execução. Dentre estas, os pesquisadores explicitaram aquelas mais comumente citadas pelos participantes, a fim de conduzir a uma apreciação mais sucinta das respostas. As atividades relativas ao autocuidado elucidadas pelos participantes foram barbear, secar o cabelo, cortar e pintar as unhas, vestir a calça, colocar os sapatos, realizar ato sexual, pintar cabelo, tomar banho, vestir, tomar remédio, beber água, comer, lavar os cabelos, abrir/abotoar fechos, abrir recipientes, escovar os dentes, andar, subir e descer escadas, entrar e sair de carros, transferir, pesar-se, entrar e sair do banheiro, andar na rua, dirigir, pegar ônibus, andar dentro do supermercado e pagar contas. As mais frequentemente mencionadas foram andar na rua, subir e descer escadas, e tomar banho.

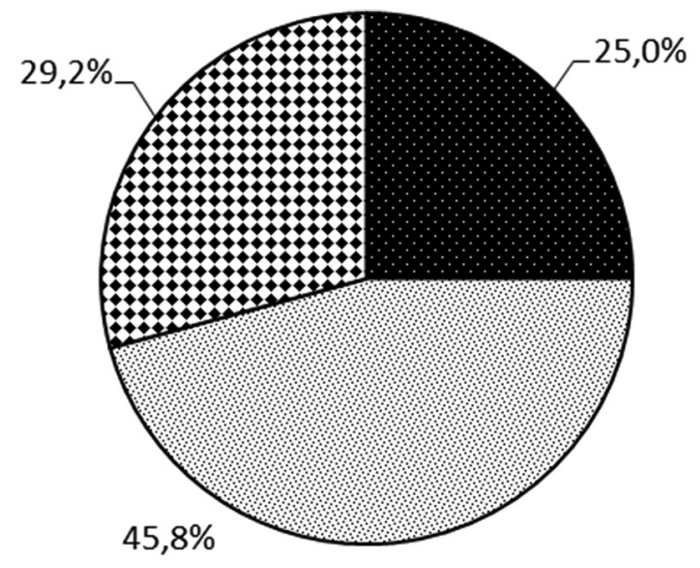

DLEVE MODERADA BGRAVE

Figura 1. Gravidade da fadiga conforme a Escala de Severidade de Fadiga ( $\mathrm{n}=27)$.
Em relação às atividades referentes à produtividade, os participantes explicitaram: trabalhar em escritório, lembrar-se de itens recentemente realizados, trabalhar em gráfica, levantar sacos de gelo, empurrar carrinhos, varrer, passar pano, limpar/arrumar a casa, lavar a casa, usar rodo, arrumar quintal, estender roupas, passar roupas, cozinhar, cuidar dos filhos e lavar banheiro. Cozinhar, limpar/arrumar a casa, e trabalhar em escritório foram as ocupaçôes mais comumente referidas.

Os participantes informaram várias atividades concernentes ao lazer, como assistir à televisão, costurar, ler, fazer artesanato, pintar, usar computador, andar de bicicleta, sair para passear, frequentar academias, fazer hidroterapia, alongar, jogar futebol, fazer caminhadas, ir ao cinema, correr, dançar, viajar, frequentar bares, ir a festas, ir à casa de amigos/familiares, e ir à praia e a shows. As atividades mais citadas foram ir à casa de amigos e familiares, dançar e sair para passear. Dentre as atividades explicitadas pelos participantes, observou-se grande diferenciação nos escores. Assim, aquelas mais mencionadas e com escores mais similares, considerando-se os três descritores da COPM, encontram-se discriminadas na Tabela 2.

Realizou-se a somatória das médias das atividades mais frequentemente enumeradas pelos participantes. As variáveis "fadiga, desempenho ocupacional e satisfação ao fazê-lo" apresentaram distribuição normal (0,50,0,86 e 0,56, respectivamente). A partir da soma das médias de tais variáveis não foi observada relação estatisticamente significante entre "fadiga" e "desempenho" na execução das atividades (r: -0,11) ou "fadiga" e "satisfação" ao desempenhar as atividades (r: -0,26). Por outro lado, constatou-se relação positiva média estatisticamente significativa entre as variáveis "desempenho" da atividade e "satisfaçáo" ao desempenhá-la (r: 0,51) (Quadro 1) (Figura 2).

Tabela 2. Porcentagem e média das atividades mais relatadas pelos participantes em relação aos descritores grau de importância, desempenho e satisfação (COPM) $(\mathrm{n}=27)$.

\begin{tabular}{lccccc}
\hline Categorias & Atividades & $\%$ & $\begin{array}{c}\text { Importância } \\
\text { (média) }\end{array}$ & $\begin{array}{c}\text { Desempenho } \\
\text { (média) }\end{array}$ & $\begin{array}{c}\text { Satisfação } \\
\text { (média) }\end{array}$ \\
\hline \multirow{4}{*}{ Autocuidado } & Andar na rua & 37,0 & 9,2 & 4,0 & 5,3 \\
& Subir e descer escadas & 25,9 & 8,1 & 4,1 & 4,1 \\
& Tomar banho & 14,8 & 10 & 4,7 & 7,5 \\
& Cortar e pintar as unhas & 14,8 & 9,5 & 3,5 & 5,0 \\
\hline \multirow{4}{*}{ Produtividade } & Cozinhar & 14,8 & 8,5 & 3,7 & 5,0 \\
& Limpar/arrumar a casa & 11,1 & 10,0 & 3,0 & 4,3 \\
& Trabalhar no escritório & 11,1 & 9,3 & 7,6 & 8,0 \\
& Passar pano & 11,1 & 10,0 & 4,0 & 5,3 \\
\hline \multirow{4}{*}{ Lazer } & Ir à casa de amigos & 18,5 & 8,8 & 5,8 & 6,2 \\
& Dançar & 14,8 & 9,0 & 6,2 & 5,7 \\
& Sair para passear & 14,8 & 8,7 & 1,7 & 3,5 \\
& Ler & 14,8 & 9,0 & 5,7 & 4,7 \\
\hline
\end{tabular}


Quadro 1. Correlação entre fadiga, desempenho e satisfação (COPM) (n=27).

\begin{tabular}{|l|l|c|c|c|}
\hline \multicolumn{2}{|c}{} & ESF & COPM desempenho & $\begin{array}{c}\text { COPM } \\
\text { satisfação }\end{array}$ \\
\hline \multirow{2}{*}{ ESF } & $\begin{array}{l}\text { Coeficiente de } \\
\text { Correlação de Pearson }\end{array}$ & 1 & -.268 & -.110 \\
\cline { 2 - 5 } & Significância (bicaudal) & & .177 & .585 \\
\hline \multirow{2}{*}{ COPM desempenho } & $\begin{array}{l}\text { Coeficiente de } \\
\text { Correlação de Pearson }\end{array}$ & -.268 & 1 &. $.518^{*}$ \\
\cline { 2 - 5 } & Significância (bicaudal) & .177 & & .006 \\
\hline \multirow{2}{*}{ COPM satisfação } & $\begin{array}{l}\text { Coeficiente de } \\
\text { Correlação de Pearson }\end{array}$ & -.110 & $.518^{*}$ & 1 \\
\cline { 2 - 5 } & Significância (bicaudal) & .585 & .006 & \\
\hline
\end{tabular}

*Correlação significante em 0,01 (bicaudal).

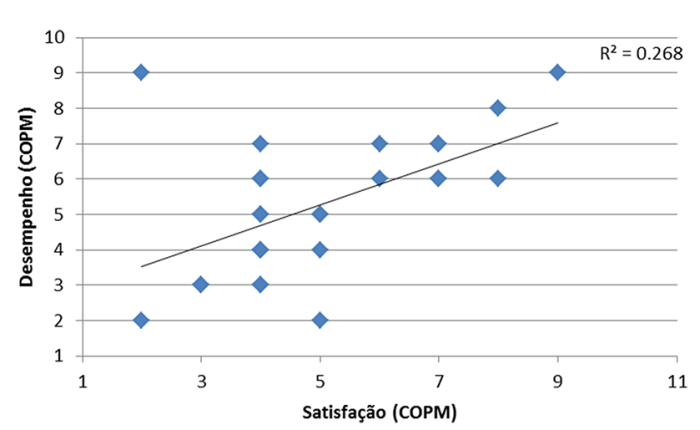

Figura 2. Correlação positiva entre desempenho e satisfação $(\mathrm{COPM})(\mathrm{n}=27)$.

\section{Discussão}

De acordo com este estudo, constatou-se que a EM afeta mais indivíduos do sexo feminino, estando a maior parte deles entre 41 e 60 anos de idade. Tais dados são corroborados por Corsi (2014), que relata que, apesar de poder ocorrer em crianças e pessoas com faixa etária mais elevada, a EM afeta principalmente adultos jovens com idades entre 20 e 50 anos. O tipo RR foi o mais observado, achado confirmado pela literatura científica (PINA, 2012).

Através da aplicaçáo da ESF, ficou constatado que a maioria dos participantes vivencia a fadiga. De fato, a fadiga é considerada comum na EM, afetando cerca de $50 \%$ a $70 \%$ dos indivíduos (LOPES et al., 2010).

Ao analisar a ESF, observou-se que, dentre as nove afirmaçôes pontuadas pelo instrumento, quatro foram assinaladas pelos participantes como áreas que sofrem maior interferência do cansaço, sendo estas: diminuição da motivação, realização de exercício, sustentação da condição física e execução de deveres e responsabilidades. Este achado confirma o exposto por Mendes (2007) de que a fadiga é referida como uma sensação subjetiva de perda de energia física e/ou mental, que conduz a um grau de incapacidade persistente.

A inexistência de correlação entre DO e fadiga observada neste estudo pode implicar em não interferência direta de tal sintoma na execuçáo das atividades pelos participantes. Isto pode levar a crer que, a despeito do cansaço caracteristicamente observado na EM, os participantes permaneceram realizando atividades. Tal achado, no entanto, contradiz a literatura científica. Stout e Finlayson (2011) afirmam que atividades diárias, papéis, rotinas, hábitos e relacionamentos são amplamente influenciados pela fadiga. Flensner et al. (2013) relatam também que a fadiga observada na EM pode ser vista como um sintoma-chave, que influencia tanto a capacidade de trabalho quanto a qualidade de vida dos indivíduos.

A não correlação entre fadiga e desempenho ocupacional observada neste estudo pode ser explicada pelo fato de haver redefiniçáo da rotina por parte dos indivíduos conforme a fadiga por eles vivenciada, fato que acarreta satisfatório desempenho frente às novas ocupaçôes que se enquadram no nível da fadiga (STOUT; FINLAYSON, 2011). Costa et al. (2005) propóem ainda que indivíduos com EM convivem com uma diversidade de sintomas diariamente, dentre os quais a fadiga, conseguindo, a despeito de sua existência, criar diversas alternativas e meios para driblar o efeito negativo desse sintoma sobre o DO. Isso é ratificado por Fragoso et al. (2010), ao explicitarem que adultos jovens com EM investiam em suas carreiras e se adaptavam às mudanças drásticas impostas por deficiências neurológicas e outros sintomas, sendo que tais alteraçóes variavam desde pequenas adaptaçôes no trabalho até importantes modificaçóes. Finalmente, a partir de enunciados dos participantes no decorrer da pesquisa, os pesquisadores deste estudo propóem explicaçôes que podem esclarecer a continuidade do desempenho nas ocupaçóes a despeito da existência da fadiga, ou 
seja: a) obrigação de se manterem produtivos a fim de cumprir funçóes impostas a eles pela sociedade ou por si próprios; b) importância de continuarem ativos, visto ser tal atitude saudável e positiva, e c) necessidade de náo se deixarem abater por um sintoma concebido como "cansaço, preguiça ou depressão” (MENDES, 2007).

Stout e Finlayson (2011) indicam que é comum que atividades de lazer sejam passivamente abandonadas por indivíduos fadigados, uma vez que as mesmas são consideradas opcionais em relação às ocupaçóes de cunho obrigatório, como as atividades de vida diária. Caso isso tivesse sido averiguado neste estudo, os autores poderiam pressupor que tal fato seria um provável motivo pelo qual não houve correlaçáo entre desempenho ocupacional - que inclui o lazer - e fadiga.

A não relação entre DO e fadiga pode também ser explicada ainda pelo modelo biopsicossocial da Classificaçáo Internacional de Funcionalidade, Incapacidade e Saúde (CIF) da Organização Mundial de Saúde. Conforme a CIF, o indivíduo não deve ser considerado apenas em função do déficit de sua saúde ou da doença que possui, mas também em relação às funçôes e estruturas de seu corpo, às atividades e participaçôes sociais que realiza ou deixa de fazer, e a barreiras ou facilitadores do ambiente que o cerca (MÂNGIA; MURAMOTO; LANCMAN, 2008). Assim, com base em tal pressuposto, apesar de a fadiga acarretar comprometimentos diversos em indivíduos com EM, suas consequências podem depender também de fatores pessoais, ambientais e sociais que influenciem sua forma de lidar com a doença.

A relação positiva observada entre as variáveis "desempenho" nas atividades e "satisfação" ao desempenhá-las pode ilustrar uma atitude positiva e otimista por parte dos participantes em relaçáo ao modo como realizam suas atividades rotineiras. Isto é pautado em um estudo conduzido por Pedro e Pais-Ribeiro (2010), através do qual se observou que há uma relação estatisticamente significativa entre otimismo e autonomia funcional em indivíduos com EM. Os autores daquele estudo enfatizam que o otimismo é uma característica estável da personalidade que é determinante em todos os acontecimentos da vida, influenciando na forma como as pessoas reagem aos problemas ou adversidades, e orientando positivamente as açôes que adotam para resolver os problemas. Assim, os otimistas, face à adversidade, adotam comportamentos positivos visando aos resultados que irão obter, traçam planos de estratégia centrados na resolução do problema, planificam a recuperação, procuram mais informação e reformulam estratégias de recuperação de forma a obter sucesso nos seus resultados, tendo facilidade para lidar com os impactos causados pela doença nas suas atividades cotidianas (PEDRO; PAIS-RIBEIRO, 2010).

Atitudes otimistas ainda são pautadas por Fournier, de Ridder e Bensing (2002 apud PEDRO; PAIS-RIBEIRO, 2010), os quais observaram relação entre a variável "otimismo" e doenças crônicas graves - como a EM - ou sintomas das mesmas, como fadiga, dor, capacidade funcional. A partir dos achados, eles concluíram que o otimismo está diretamente relacionado a uma melhor adaptação dos doentes à sua situação e, com a redução da ansiedade, além de auxiliar na melhora da funcionalidade física e psíquica em doentes com EM, estes tinham melhores comportamentos de autocuidado.

\section{Considerações finais}

O estudo demonstrou que há relação diretamente positiva entre o desempenho ocupacional e a satisfação na realização das atividades por parte dos participantes. Considerando-se que o desempenho é formado pela associação de itens diversos, pode-se compreender que os sintomas gerados pela EM podem ser contornados a partir de fatores pessoais utilizados por cada paciente, o qual cria estratégias de adaptação para lidar com suas limitaçóes e dificuldades. Ressalta-se que os fatores pessoais e as atitudes otimistas e positivas podem surtir efeito na satisfação dos participantes, que passam a se sentir bem com o modo nem sempre ideal como realizam as atividades. Eles compreendem a gravidade da doença e o fato de que, depois de cada surto, as incapacidades podem se tornar ainda mais graves, o que os faz valorizar as capacidades que possuem naquele momento para realização de suas atividades rotineiras.

Ao afirmar que os indivíduos que compuseram a amostra desta pesquisa participam na execução de atividades a despeito do nível de fadiga por eles exibido, os autores chamam a atenção sobre a subjetividade de tal afirmativa devido ao número reduzido da amostra e ao fato de os participantes terem apenas sido questionados sobre sua percepção em relação a tal fato, sem ter havido uma mensuração comprobatória mais fidedigna. Assim, considera-se necessária a condução de futuros estudos que utilizem instrumentos de cunho quantitativo para se averiguar a veracidade de tal afirmativa com o mínimo de possibilidade de margens de erro.

A atitude otimista por parte dos participantes observada através da relação positiva entre o 
desempenho nas atividades e a satisfação ao fazê-lo pautou-se na relaçáo entre as respostas de uma mesma avaliação - a saber, COPM -, não representando, necessariamente, aspectos emocionais dos indivíduos. Sugere-se, portanto, aprimorar a discussão de tal tópico com foco nos resultados observados neste estudo.

A fim de dar continuidade a este estudo, recomenda-se que sejam conduzidas pesquisas que avaliem a fadiga de forma mais abrangente e de acordo com o parecer do sujeito, pois, pela avaliaçáo através do instrumento utilizado neste estudo, tal variável foi averiguada apenas quantitativamente. Instrumentos desse porte, associadamente a outras avaliaçóes, podem ser utilizados com o objetivo de coletar informaçóes que posteriormente permitam a elaboração de programas para orientação individualizada que visem a averiguar não só a fadiga, mas outros fatores a esta associados.

Sugere-se a realização de estudos futuros com indivíduos que exibam fadiga devido a patologias nas quais tal sintoma é característico - como Síndrome Pós-Pólio, DPOC, Esclerose Lateral Amiotrófica, dentre outras -, com o objetivo de verificar a existência de correlação entre tal sintoma e o desempenho ocupacional em tais doenças, sobretudo de acordo com o grau da fadiga.

Propóe-se também que sejam desenvolvidas pesquisas que constatem outras variáveis que possam explicar a não correlação entre desempenho ocupacional e fadiga, como aceitabilidade da doença, qualidade de vida, suporte familiar e comunitário, religiáo, uso de equipamentos de autoajuda, acessibilidade domiciliar e comunitária, etc.

Pressupóe-se que o número reduzido de participantes possa ter influenciado os achados desta pesquisa. Assim, é interessante que pesquisas com o tema deste estudo sejam conduzidas com um maior número de indivíduos com esclerose múltipla, a fim de averiguar se haverá ou não correlação entre fadiga e DO com maior fidedignidade.

Os resultados deste estudo demonstram aplicabilidade para a prática clínica visto possibilitar ao profissional se pautar na relaçáo entre desempenho ocupacional e satisfação ao elaborar objetivos na abordagem preventiva ou reabilitadora.

\section{Referências}

BARROSO, S. M. B. et al. Dificuldades na avaliação neuropsicológica de pacientes com esclerose múltipla. Revista Neurociências, São Paulo, v. 21, n. 1, p. 53-59, 2013.
BRASIL. Ministério da Saúde. Resolução No 466, de 12 de Dezembro de 2012. Diário Oficial [da] Uniāo, Brasília, DF, 13 jun. 2013. Seção 1, p. 59. Disponível em: <http://conselho.saude.gov.br/resolucoes/2012/ Reso466.pdf>. Acesso em: 05 mar. 2014.

COPPERMAN, L. F.; FORWELL, S. J.; HUGOS, L. Doenças neurodegenerativas. In: TROMBLY, C. A.; RADOMSKI, M. V. Terapia Ocupacional para disfunçóes fisicas. São Paulo: Santos, 2008. p. 885-908.

CORSI, G. Doença e crença religiosa: as diversas formas de significar as perdas na esclerose múltipla. Saberes em Ação, Mato Grosso do Sul, v. 2, n. 3, p. 40-55, 2014.

COSTA, C. C. R. et al. O adoecimento do portador de esclerose múltipla. Revista Brasileira em Promoçāo da Saúde, Maruípe, v. 18, n. 3, p. 117-124, 2005. http:// dx.doi.org/10.5020/18061230.2005.p117.

FLENSNER, G. et al. Work capacity and health-related quality of life among individuals with multiple sclerosis reduced by fatigue: a cross-sectional study. BMC Public Health, London, v. 13, p. 1-10, 2013. http://dx.doi. org/10.1186/1471-2458-13-224. PMid:23497281.

FRAGOSO, Y. D. et al. The effect of multiple sclerosis on the professional life of a group of Brazilian patients. Arquivos de Neuro-Psiquiatria, São Paulo, v. 68, n. 6, p. 914-917, 2010.

HOFFMANN, P.; DYNIEWICZ, A. M. A terapia ocupacional na esclerose múltipla: conhecendo e convivendo para intervir. Cogitare Enfermagem, Curitiba, v. 14, n. 2, p. 285-293, 2009. http://dx.doi.org/10.5380/ ce.v14i2.15620.

KOCHAHNN, R. et al. The mini mental state examination. Review of cutoff points adjusted for schooling in a large Southern Brazil sample. Dementia \& Neuropsychologia, São Paulo, v. 4, n. 1, p. 35-41, 2010.

LOPES, K. N. et al. Limitação funcional, fadiga e qualidade de vida na forma progressiva primária da esclerose múltipla. Revista Neurociências, Sáo Paulo, v. 18, n. 1, p. 13-17, 2010.

MAGALHÂES, L. C.; MAGALHÂES, L. V.; CARDOSO, A. A. Medida canadense de desempenho ocupacional (COPM). Belo Horizonte: Editora UFMG, 2009.

MÂNGIA, E. F.; MURAMOTO, M. T.; LANCMAN, S. Classificação Internacional de Funcionalidade, Incapacidade e Saúde (CIF): processo de elaboração e debate sobre a questão da incapacidade. Revista de Terapia Ocupacional da Universidade de São Paulo, São Paulo, v. 19, n. 2, p. 121-130, 2008.

MENDES, M. F. Fadiga na esclerose múltipla. Revista Neurociências, São Paulo, v. 15, p. 181, 2007.

MENDES, M. F. et al. Fadiga na forma remitente recorrente da esclerose múltipla. Arquivos de Neuro-Psiquiatria, Sáo Paulo, v. 58, n. 2-B, p. 471-475, 2000.

MESQUITA, B. S. Análise da influência da abordagem fisioterapêutica sobre a fadiga em individuos com esclerose 
múltipla: uma revisão sistemática. 2013. 24 f. Monografia (Bacharelado em Fisioterapia) - Universidade de Brasília, Brasília, 2013.

MILO, R.; KAHANA, E. Multiple sclerosis: geoepidemiology, genetics and the environment. Autoimmunity Reviews, Netherlands, v. 9, n. 5, p. A387-A394, 2010. http://dx.doi.org/10.1016/j.autrev.2009.11.010. PMid:19932200.

NOGUEIRA, L. A. C. et al. The effect of functional limitations and fatigue on the quality of life in people with Multiple Sclerosis. Arquivos de Neuro-Psiquiatria, São Paulo, v. 67, n. 3-B, p. 812-817, 2009.

OLIVEIRA, A. C. F. R. et al. Caracterização e queixas relacionadas ao desempenho ocupacional: consideraçóes de indivíduos com esclerose múltipla. Revista de Terapia Ocupacional da Universidade de São Paulo, São Paulo, v. 24, n. 2, p. 112-20, 2013.

PEDRO, L.; PAIS-RIBEIRO, J. L. P. Implicaçóes da situação profissional na qualidade de vida em indivíduos com esclerose múltipla. In: SIMPÓSIO NACIONAL DE INVESTIGAÇÃO EM PSICOLOGIA, 7., 2010,
Braga. Anais... Braga: Universidade do Minho, 2010. p. 1286-1294.

PINA, L. I. G. Esclerose múltipla: terapêutica com ITF $\beta$. 2012. 57 f. Tese (Mestrado em Ciências Farmacêuticas) - Universidade Fernando Pessoa, Porto, 2012.

QUINTANILHA, R. S.; LIMA, L. R. Avaliação da qualidade de vida em portadores de Esclerose Múltipla. Revista de Enfermagem UFPE, Recife, v. 4, n. 1, p. 153161, 2010.

SANTOS, F. L. V. et al. A vivência do cônjuge/companheiro de portador de esclerose múltipla. Revista de Enfermagem UFRJ, Rio de Janeiro, v. 18, n. 2, p. 229234, 2010.

SILVA, D. F.; NASCIMENTO, V. M. S. Esclerose múltipla: imunopatologia, diagnóstico e tratamento - artigo de revisão. Interfaces Cientificas - Saúde e Ambiente, Aracaju, v. 2, n. 3, p. 81-90, 2014.

STOUT, K.; FINLAYSON, M. Management in chronic illness. OT Practice, Bethesda, v. 24, p. 17-26, 2011.

\section{Contribuição dos Autores}

Valéria Sousa de Andrade: responsável pela orientação e revisão do texto; Mayara Mirella Araújo Seabra e Isabella Elias de Moura Ramos: responsáveis pela concepção e redação do texto e das fontes. Todas as autoras aprovaram a versão final do texto.

\section{Notas}

${ }^{1} \mathrm{O}$ material envolveu seres humanos, tendo cumprido os procedimentos éticos vigentes. É parte de uma Iniciação Científica em Terapia Ocupacional do Grupo de Estudo e Pesquisa em Esclerose Múltipla (GEPEM). O texto foi apresentado na XX Jornada de Iniciaçáo Científica da Universidade Federal do Triângulo Mineiro (UFTM) em 2012. 